\title{
Optimal Workload of Wingate Test: A Comparison Between Normal and Minor Overfat Young Adults
}

\author{
Kui Lu ${ }^{1}$, Binh Quach ${ }^{2}$, Pak Kwong Chung ${ }^{1,2}$ and Tom K. Tong*,1,2 \\ ${ }^{1}$ Dr. Stephen Hui Research Centre for Physical Recreation and Wellness, Hong Kong Baptist University and \\ ${ }^{2}$ Department of Physical Education, Hong Kong Baptist University, China
}

\begin{abstract}
We compared the optimal workload of Wingate test (WAnT), which was assigned according to total body mass (TBM), between two groups of eight young male adults. The leg anaerobic power and fat free mass of these two groups were comparable while their percent body fat was either within (NOR) or above normal range (OF). The optimal WAnT workload was derived individually from the polynomial regression of power-workload against of 75, 100, 110, 125 and 140 g.kg ${ }^{-1}$ TBM. It was found that the maximum power elicited from the various workloads in the WAnT was not different $(\mathrm{P}>0.05)$ between NOR and OF groups. Further, there was no difference in the optimal workload between the two groups (NOR: $120.7 \pm 13.6$; OF: $114.0 \pm 7.2 \mathrm{~g} . \mathrm{kg}^{-1} \mathrm{TBM}, \mathrm{P}>0.05$ ). The findings suggest that the counting of fat mass in workload assignment in the WAnT may not affect the maximum power output in minor overfat adults.
\end{abstract}

Key Words: Wingate test, Fat mass, Workload, Anaerobic power, Young adults.

\section{INTRODUCTION}

Inbar et al. [1] developed the Wingate anaerobic test (WAnT) in leg by assigning a workload at $75 \mathrm{~g}^{\mathrm{kg}} \mathrm{kg}^{-1}$ total body mass (TBM) of the subjects. Van Praagh and Franca [2] indicated that there was a methodological shortcoming in the WAnT as the workload assigned was based on the TBM, which did not take into account the active muscle mass. In human, the TBM, which is composed of several components including the mass of fat, bone, viscera and muscle, can be generally divided into fat mass (FM) and fat free mass (FFM). It is believed that the fat tissues, mainly the storage fats, in the body do not contribute to one's anaerobic power.

Üçok et al. [3] demonstrated a significant increase in peak power among the young males in the WAnT when using a workload of $100-110 \mathrm{~g} \cdot \mathrm{kg}^{-1}$ lean body mass. They suggested that lean body mass would be a more appropriate reference in deriving the workload in the WAnT for young males. However, the influence on leg power output by counting the FM into the workload used in the WAnT was not reported. A recent study [4] reported that the assignment of the WAnT workload according to FFM (86 g. $\mathrm{kg}^{-1}$ FFM) could develop the peak power (PP) and mean power (MP) equivalent to those obtained from the original workload of $75 \mathrm{~g} \cdot \mathrm{kg}^{-1} \mathrm{TBM}$ [1] in male young adults with normal percent body fat (\%BF). When the WAnT workload of $86 \mathrm{~g} . \mathrm{kg}^{-1}$ FFM, which the FM was not counted in, was applied to other subjects with homogeneous physical characteristics except greater FM (minor overfat) and TBM, the PP and MP resulting from the original workload of $75 \mathrm{~g} . \mathrm{kg}^{-1}$ TBM were decreased. Such findings did not agree with the previous notion of Üçok et al. [3]. It appeared that the counting of FM in the

*Address correspondence to this author at the Research Center for Physical Recreation and Wellness, NAB210, L2, David C. Lam Bldg. Shaw Campus, Hong Kong Baptist University, Renfrew Rd., Kowloon Tong, Hong Kong, China; Tel: (852) 3411 5758; Fax: (852) 3411 5756;

E-mail: tongkk@hkbu.edu.hk workload assignment may not affect the WAnT performance in minor overfat young adults. However, the previous study did not compare the optimal workload of the WAnT, which was derived according to TBM, between the two groups of subjects. For the counting of FM in the load assignment was a negative factor to influence the WAnT performance in the minor overfat subjects, their optimal load of the WAnT should be lower compared with that of their counterpart with normal \%BF. The purpose of this study was to compare the optimal load of the WAnT between two groups of young male adults with \%BF either within (NOR) or above normal range $(\mathrm{OF})$.

\section{METHOD}

\section{Subjects}

Sixteen male undergraduate Physical Education students, who participated regularly in various sports training including soccer and rugby, were recruited for this study. Eight subjects in the NOR group were with \%BF below the cutoff of $15.0 \%$ that was defined as "Average" in young adults either normal or athletic trained $[5,6]$. The other subjects in the OF groups were with \% BF above the cutoff. Nevertheless, the FFM, and the anaerobic power and fatigability in knee extensors and flexors of both groups were not different (Table 1). After being fully informed of the experimental procedures and possible discomfort associated with the exercise test, subjects gave their written consent. Ethical approval for this study was obtained from the Committee on the Use of Human and Animal Subjects in Teaching and Research of Hong Kong Baptist University. The physical characteristics of the subjects in both groups are presented in Table 1.

\section{Procedures}

\section{Research Design}

Optimal WAnT workload for each subject in both NOR and OF groups was derived individually from the polynomial 
regression of power-workload. The regression was fitted by curve drawn according to the maximum power and workload measured during WAnT against five discrete loads of 75 , $100,110,125$ and 140 g.kg ${ }^{-1}$ TBM. The workload corresponding to the highest value of maximum power in the curve was defined as the optimal WAnT workload. For examining the reliability of maximum power elicited by the assigned workloads, each WAnT was repeated twice.

Table 1. Physical Characteristics of Normal (NOR) and Minor Overfat (OF) Subjects

\begin{tabular}{|c|c|c|c|c|c|c|c|}
\hline & & & $\mathbf{R}(\mathbf{I}$ & & & $\mathrm{F}(\mathbf{n}$ & \\
\hline \multicolumn{2}{|c|}{ Age (years) } & 21.6 & \pm & 0.7 & 22 & \pm & 1.3 \\
\hline \multicolumn{2}{|c|}{ Height $(\mathrm{cm})$} & 175 & \pm & 3.8 & 176.7 & \pm & 4.8 \\
\hline \multicolumn{2}{|c|}{ Weight (kg) } & 63.2 & \pm & 4.4 & $69.4^{*}$ & \pm & 7.1 \\
\hline \multicolumn{2}{|c|}{$\% \mathrm{BF}(\%)$} & 12.7 & \pm & 0.9 & $19.6^{*}$ & \pm & 2.7 \\
\hline \multicolumn{2}{|c|}{ FFM (kg) } & 55.2 & \pm & 3.9 & 55.8 & \pm & 6.8 \\
\hline Isokinetic & MPO (W) & 617 & \pm & 103 & 660.4 & \pm & 100 \\
\hline Test in Legs & FI (\%) & 41.4 & \pm & 16 & 44.4 & \pm & 11 \\
\hline
\end{tabular}

Data are means $\pm \mathrm{SD}$. \%BF is percent body fat. FFM is free fat mass. MPO is mean power output. FI is fatigue index. * Different from corresponding normal group $\mathrm{P}<0.05$.

\section{Preliminary Testing}

Prior to the experimental trials, isokinetic leg test and body composition assessment were conducted. In the isokinetic test, maximal power in knee extensors and flexors of both legs were measured by following the protocol previously described [7]. Briefly, the subject was stabilized on an isokinetic dynamometer machine (Humac Norm, CSMI, MA, US). The range of motion in knee was set at $120^{\circ}\left(0^{\circ}\right.$ refers to full extension). The angular velocity was at $240^{\circ} \cdot \mathrm{s}^{-1}$ for both flexion and extension. The subject performed extension-flexion reciprocally at the maximal effort to reach 30 repetitions. The integrated anaerobic power in legs was the sum of the medium power of the extensors and flexors of both legs. The fatigue index was the $\%$ decline in the power between the initial and the final repetition.

For assessing body composition, bio-impedance measurement was performed using a leg-to-leg BIA body fat analyzer (Tanita, TBF-410, Tanita Corp., Tokyo, Japan). The measuring procedure was described previously [8]. Briefly, subjects were asked to stand barefoot on the metal sole plates of the machine. Gender and height details were entered manually into the system via a keyboard. \%BF, FM, and FFM were estimated using the standard built-in prediction equations for young adults. All the measurements were taken place in the morning before breakfast with subjects fasting for at least eight hours.

\section{Familiarization}

At least two familiarization trials with loading identical to the experimental trials were undertaken to familiarize the subject with the WAnT protocol and the electromagnetically braked ergometer (17985, Excalibur, Lode, Groningen, Netherlands) that would be used in subsequent tests. Seat and handlebar positions were adjusted and kept constant for each individual subjects during the course of the study.

\section{Wingate Anaerobic Test (WAnT)}

Prior to each trial, the subject refrained from eating for at least two hours and from participation in strenuous physical activity for at least 24 hours. All trials were scheduled to occur at the same time of day and were separated by a minimum of 3 days.

Prior to the WAnT, a standard 5-min warm-up exercise at $50 \mathrm{~W}$ was performed on the Lode ergometer. After the subsequent 5-min leg stretching exercise on floor, the subject sprinted twice on the ergometer at 50-75\% of the prescribed testing load for $15 \mathrm{~s}$ with a recovery interval of 30-s. After completion of the warm-up, the subject continued to pedal at 60 rev. $\min ^{-1}$ with minimal resistance for $1 \mathrm{~min}$ and started to accelerate the pedal frequency. Following a 3-s countdown, the load was immediately applied and the subject was verbally encouraged to pedal as fast as possible in the subsequent $30 \mathrm{~s}$. During the test, the subject was required to remain seated. After completion of the test, the subject continued to cycle at a light load for recovery. In the WAnT, PP was the instantaneous highest power output; MP was the average power output. The ergometer was interfaced with a computer loaded with software (Lode ergometry manager) for manipulating the testing load and measuring the PP and MP.

\section{Statistical Analysis}

The repeatability coefficient of Bland-Altman plot and intra-class reliability coefficient were calculated for determining the reliability of the PP and MP. Interactive effect of WAnT workloads $\left(75,100,110,125\right.$ and 140 g.kg ${ }^{-1}$ TBM) and groups (NOR \& OF) on the PP and MP were examined using two-way ANOVA with repeated measure in one factor. Post-hoc analyses with Newman-keuls were performed when main effects of ANOVA were significant. Independent $t$-test was applied to assess the difference in optimal WAnT workload between NOR and OF groups. All tests for statistical significance were standardized at an alpha level of $P<0.05$, and all results are expressed as the mean \pm SD.

\section{RESULTS}

For the reliability of the PP and MP, the repeatability coefficient was $6.7 \%$ and $14.8 \%$; the mean difference between the first and repeated trials was $4.9 \pm 40.5 \mathrm{~W}$ and $7.0 \pm 40.3$ $\mathrm{W}$, respectively. The intra-class reliability coefficient for the PP and MP were 0.99 and 0.97 , respectively. For data analyses, the average value of PP and MP recorded in two identical trials of each workload were calculated.

Fig. (1) shows the polynomial relationships between workload and the two variables of maximum power in WAnT in NOR and OF groups. It was found in both groups that the PP and MP increased from the initial workload of 75 g.kg ${ }^{-1}$ TBM and peaked approximately at the workload of $110 \mathrm{~g} \cdot \mathrm{kg}^{-1} \mathrm{TBM}(\mathrm{P}<0.05)$. With further increase in workload, the PP and MP decreased but only the decrease in MP was found statistically significant $(\mathrm{P}<0.05)$. The alterations in the $\mathrm{PP}$ and MP in WAnT resulting from the variation in workload are similar in both NOR and OF groups $(\mathrm{P}>0.05)$. 


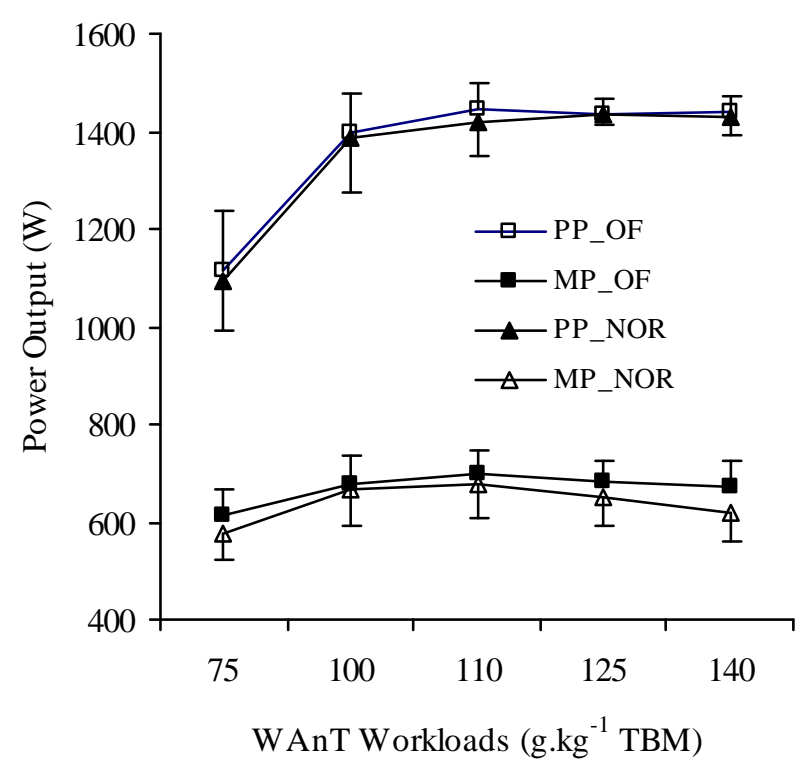

Fig. (1). The polynomial regressions of workload plotted against peak power (PP) and mean power (MP) in the WAnT for NOR and OF groups are shown. Statistical differences in the PP and MP across workloads are described in text.

The optimal workload in the WAnT for each subject was computed from the polynomial regressions of workload-PP and workload-MP (Fig. 2). The workload corresponding to the highest value of power output in each regression curve was the optimal workload in the WAnT. It was found that the mean values of the optimal WAnT workload for PP (Fig. 1) in the NOR and OF group were $127.8 \pm 14.8 \mathrm{~g} . \mathrm{kg}^{-1}$ TBM and $117.0 \pm 7.3 \mathrm{~g} . \mathrm{kg}^{-1} \mathrm{TBM}$, respectively. The difference between the two workloads is not significant $(\mathrm{P}>0.05)$. Similar results were found in the MP (Fig. 1). The difference in the optimal WAnT workload for MP between the NOR $\left(113.6 \pm 8.0 \mathrm{~g} . \mathrm{kg}^{-1} \mathrm{TBM}\right)$ and $\mathrm{OF}\left(111.0 \pm 6.2 \mathrm{~g} . \mathrm{kg}^{-1} \mathrm{TBM}\right)$ groups was also not significant $(\mathrm{P}>0.05)$.

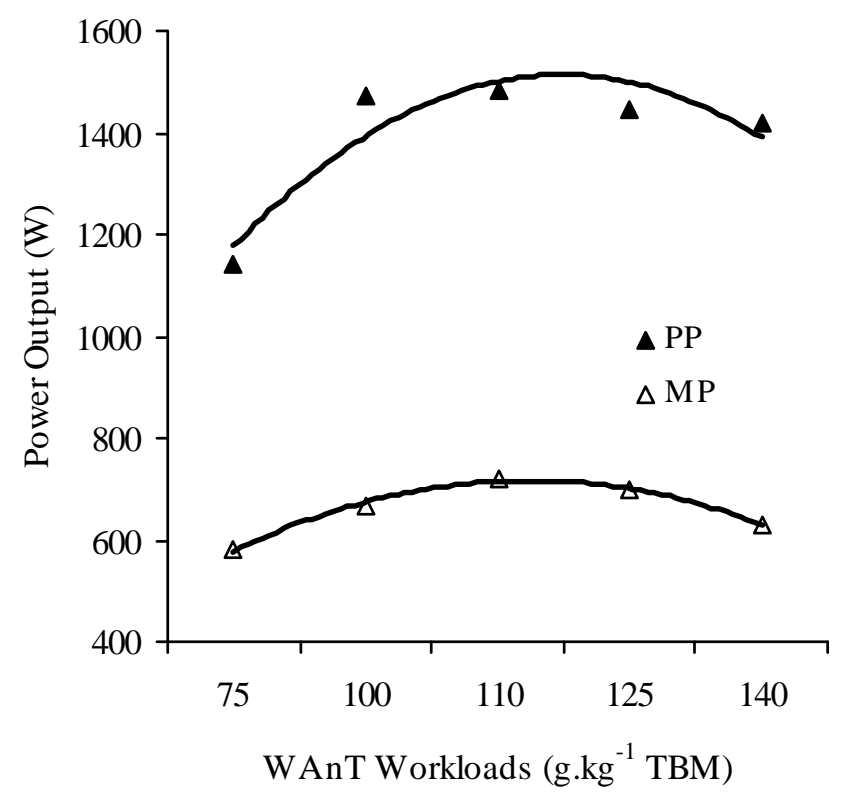

Fig. (2). The polynomial regressions of workload plotted against PP $\left(y=-54.429 x^{2}+379.87 x+851\right)$ and MP $\left(y=-27.786 x^{2}+179.61 x\right.$ +426.6 ) in the WAnT for a typical subject are shown.

\section{DISCUSSION}

The present study shows that the optimal workload in the WAnT for both NOR and OF groups are greater than the original workload of 75 g. $\mathrm{kg}^{-1}$ TBM. Further, there is no significant difference in the optimal WAnT workload between the NOR and OF groups. It appears that the counting of FM in load assignment does not affect the WAnT performance in minor overfat young adults.

As the power output during the WAnT depends upon the pedaling workload and velocity while the maximal pedaling velocity is limited by factors independent of leg anaerobic capacity, the assignment of the workload becomes crucial in the test validity [9]. Various studies reported that a load of $75 \mathrm{~g} . \mathrm{kg}^{-1}$ TBM commonly used in the WAnT was inadequate to obtain the highest power output $[9,10]$. Bar-Or [11] reported that for obtaining the highest values in PP and MP in the WAnT, the testing load should be set at $90 \mathrm{~g} \cdot \mathrm{kg}^{-1} \mathrm{TBM}$ for adults while athletes, especially the anaerobic type, should be at 100 g.kg ${ }^{-1}$ TBM. Such setting was further agreed by the subsequent studies when the workload of $105 \mathrm{~g} \cdot \mathrm{kg}^{-1}$ TBM was shown as most appropriate in trained persons for obtaining maximal power output in the WAnT $[12,13]$. In the present study, all subjects have participated in regular sports training. The mean value of optimal WAnT workload found in these subjects was $117.3 \pm 11.2{\mathrm{~g} . \mathrm{kg}^{-1}}^{\mathrm{T}} \mathrm{TBM}$. This result provides further evidence to support previous findings that the WAnT workload for eliciting the highest values in PP and MP in trained young adults should be greater than the original value of $75 \mathrm{~g}_{\mathrm{gg}} \mathrm{kg}^{-1}$ TBM and it may be close to 117 g.kg ${ }^{-1}$ TBM.

It is generally agreed that the fat compartment of body composition would not contribute to the anaerobic power output. Rather, the body fatness was negatively correlated to the anaerobic capacity measured in the WAnT in various populations including athletes and adolescents [14-16]. On the other hand, Blimkie et al. [17] found that the PP and MP in arm were related to arm volume and lean body mass in adolescents. Similar correlations were also observed in leg [18]. In the WAnT, a loading of $75 \mathrm{~g} \cdot \mathrm{kg}^{-1} \mathrm{TBM}$ is used in the general healthy population, which is based on an assumption that these people have a similar relationship between muscle mass and TBM. However, in population with abnormal muscle mass to TBM ratio such as individuals with muscle atrophy and excessive body fat may invalidate the workload assignment in the WAnT based on the TBM [19].

Nevertheless, in comparison with the performance in WAnT between NOR and OF groups, we found that there was no significant difference in PP and MP resulting from the various workloads. The discrepancy in the optimal WAnT workload computed from the workload-power output regression was also not significant between the two groups. These findings show that the burden of counting the FM in WAnT workload assignment in minor overfat young adults is not sufficient to lead to impairment in the maximum power output. In our recent study, it was found that the PP and MP resulting from the workload of $75 \mathrm{~g}^{\mathrm{kg}} \mathrm{kg}^{-1} \mathrm{TBM}$ decreased in minor overfat subjects when the FM was not counted in the load assignment [4]. The decrease in the maximum power output was correlated to the $\% \mathrm{BF}$. When incorporating the previous findings into the present study, 
we deem that the negative influence of counting the FM in WAnT workload assignment on the maximum power output in minor overfat young adults is minimal. However, the notion should be interpreted with caution as the subjects in the $\mathrm{OF}$ group were trained. Moreover, their \%BF, unlike that of obese persons, was just a little above the cutoff of average value, by which the findings may not be generalized to the adults with higher \%BF. Further studies to address all these concerns are recommended.

In conclusion, the optimal workload in WAnT for assessing maximum power output in young male adults was approximately 117 g. $\mathrm{kg}^{-1}$ TBM. There was no significant difference in the optimal WAnT workload between normal and minor overfat young adults. In light of the current findings and those in our previous study [4], it appears that the counting of FM in the WAnT workload assignment may not be a negative factor to influence the maximum power output in minor overfat adults.

\section{REFERENCE}

[1] Inbar O, Ayalon A, Bar-Or O. Relationship between tests of anaerobic capacity and power. Isr J Med Sci 1974; 10: 290.

[2] Van Praagh E, Franca NM. In: Van Praagh E, Ed. Pediatric Anaerobic Performance. Illinois, Human Kinetics 1998; 155-89.

[3] Üçok K, Gökbel H, Okudan N. The load for the Wingate test: according to the body weight or lean body mass. Eur J Gen Med 2005; $2: 10-3$

[4] Tong TK, Lu K, Chung PK, Quach B. The influence of fat mass on evaluating leg anaerobic power with the Wingate test in minor overfat young adults. J Exerc Sci Fit 2008; In Press.

[5] Lohman TG. Advances in body composition assessment. Current Issues in Exercise Science. Illinois: Human Kinetics 1992.

[6] McArdle WD, Katch FI, Katch VL. Exercise Physiology-Energy, Nutrition, and Human Performance. 5th ed. Baltimore: Lippincott Williams \& Wilkins 2001.
[7] Tsaklis P. Isokinetic Evaluation of the knee extensors and flexors anaerobic capacity. Isokinet Exerc Sci 2002; 10: 69-72.

[8] Lu K, Quach B, Tong TK, Lau PWC. Validation of leg-to-leg bioimpedance analysis for assessing body composition in obese Chinese children. J Exerc Sci Fit 2003; 1: 97-103.

[9] Dotan R, Bar-Or O. Load optimization for the Wingate Anaerobic Test. Eur J Appl Physiol Occup Physiol 1983; 51: 409-17.

[10] Patton JF, Murphy MM, Frederick FA. Maximal power outputs during the Wingate anaerobic test. Int J Sports Med 1985; 6: 82-5.

[11] Bar-Or O. The Wingate anaerobic test. An update on methodology, reliability and validity. Sports Med 1987; 4: 381-94.

[12] Hermina W. The Effects of different resistances on peak power during the Wingate anaerobic test. MS. diss., Dept. of Nutrition and Exercise Sciences, Oregon State Univ 1999.

[13] Sidner AB. The effects of high resistances on peak power output and total mechanical work during short-duration high intensity exercise in the elite female athlete. MS. diss., Dept. of Nutrition and Exercise Sciences, Oregon State Univ 1998.

[14] Lafortuna CL, Agosti F, Marinone PG, Marazzi N, Sartorio A. The relationship between body composition and muscle power output in men and women with obesity. J Endocrinol Invest 2004; 27: 85461.

[15] Pilis W, Wojtyna J, Langfor J, et al. Relationships between sport results, somatic variables and anaerobic power in elite weight lifters. Biol Sport 1997; 14: 275-81.

[16] Armstrong N, Welsman JR, Williams CA, Kirby BJ. Longitudinal changes in young people's short-term power output. Med Sci Sports Exerc 2000; 32: 1140-5.

[17] Blimkie CJ, Roache P, Hay JT, Bar-Or O. Anaerobic power of arms in teenage boys and girls: relationship to lean tissue. Eur J Appl Physiol Occup Physiol 1988; 57: 677-83.

[18] Van Praagh E, Fellmann N, Bedu M, Falgairette G, Coudert J. Gender difference in the relationship of anaerobic power output. Pediatr Exerc Sci 1990; 2: 336-48.

[19] Van ME, Schoeber N, Calvert RE, Bar-or O. Optimization of force in the Wingate test for children with a neuromuscular disease. Med Sci Sports Exerc 1996; 28: 1087-92. 